\title{
Emerging roles of exosomes in cancer invasion and metastasis
}

\author{
Young Hwa Soung ${ }^{1}$, Thalia Nguyen ${ }^{2}$, Hans Cao ${ }^{2}$, Janet Lee ${ }^{2}$ E Jun Chung ${ }^{1, *}$ \\ ${ }^{1}$ Department of Pathology, Stony Brook Medicine, Stony Brook, NY 11794, ${ }^{2}$ Department of Physiology, University of Oklahoma Health \\ Sciences Center, Oklahoma City, OK 73034, USA
}

\begin{abstract}
Recent evidence has indicated that nano-sized vesicles called "exosomes" mediate the interaction between cancer cells and their microenvironment and play a critical role in the development of cancers. Exosomes contain cargo consisting of proteins, lipids, mRNAs, and microRNAs that can be delivered to different types of cells in nascent as well as distant locations. Cancer cell-derived exosomes (CCEs) have been identified in body fluids such as urine, plasma, and saliva from patients with cancer. Although their content depends on tumor type and stage, CCEs merit consideration as prognostic and diagnostic markers, as vehicles for drug delivery, and as potential therapeutic targets because they could transport various oncogenic elements. In this review, we summarize recent advances regarding the role of CCEs in cancer invasion and metastasis, as well as its potential clinical applications. [BMB Reports 2016; 49(1): 18-25]
\end{abstract}

\section{INTRODUCTION}

The secretion of extracellular vesicles (EVs) is a well-conserved evolutionary process that occurs from prokaryotic cells to all eukaryotes (1). EVs are categorized by various nomenclatures based on their size, intracellular origin, and mode of formation (i.e., exosomes, ectosomes, and apoptotic bodies). Ectosomes are shedding microvesicles directly derived from the plasma membrane, whereas dying cells produce apoptotic bodies to prevent leakage of their toxic cellular components (2). Initially, it was thought that all EVs originate directly from the plasma membrane by budding; however, exosomes are distinct from other EVs with respect to their origin, size, function, and composition. The term "exosomes" was first proposed in the late 1980s to describe small (30- to 100-nm) vesicles of endosomal origin that are released during reticulocyte maturation and are rich in 5'-nucleotidase activity (3). Invagination of the endo-

*Corresponding author. Tel: +631-444-3000; Fax: +631-444-3424; E-mail: jun.chung@stonybrookmedicine.edu

http://dx.doi.org/10.5483/BMBRep.2016.49.1.239

Received 18 November 2015

Keywords: Exosomes, Invasion, Metastasis, Drug resistance, Biomarkers, Therapeutic application somal membrane induces the formation of small vesicles, which in turn leads to the formation of multivesicular bodies (MVBs) and subsequent release of these vesicles through fusion with the plasma membrane. Subsequent studies have revealed that exosome secretion into extracellular media such as blood, saliva, and urine occurs in multiple cell types, including cancer cells (4).

In recent years, these nanoscale vesicles have garnered a lot of attention from cancer researchers because they contain bioactive molecules such as lipids, proteins, and nucleic acids that can be transferred from primary tumors to various organs and tissues $(1,5,6)$. Mounting evidence supports the role of exosomes in multiple aspects of cancer processes, such as transforming neighboring cells within the tumor microenvironment and enhancing the invasiveness and drug-resistant potential of other tumor cells through the transport of oncogenic elements (5-7). Therefore, exosomes can create an ideal milieu in which primary tumor cells can interact with neighboring cells for successful growth and metastasis. In this review, we will focus on issues regarding 1) the way cancer cells organize and sort exosomes differently from normal cells; 2) the role of oncogenic elements of CCEs in invasion, metastasis, and drug resistance; and 3) how our current knowledge about CCEs can contribute to the development of novel biomarkers and therapeutic interventions.

\section{MECHANISMS OF BIOGENESIS, SECRETION, AND UPTAKE OF EXOSOMES}

Exosomes are formed as intraluminal vesicles (ILVs) through the endocytic pathway and budding into early endosomes and multivesicular bodies (MVBs). MVBs are defined by the presence of small, cytosol-containing ILVs that are formed from the limiting membrane of late endosomes (8). The fate of MVBs is either fusion with lysosomes for cargo degradation or fusion with the plasma membrane for releasing ILVs to the extracellular milieu as exosomes (9) (Fig. 1).

One of the well-known mechanisms that describe the formation of ILVs and cargo sorting involves the endosomal sorting complex required for transport (ESCRT) machinery (10). ESCRT comprises four complexes (0, I, II, and III) plus associated proteins (i.e., VPS4, VTA1, ALIX, and TSG101). ESCRT 0 involves cargo clustering by recognizing and sequestering ubiquitinated transmembrane proteins in the endosomal mem- 


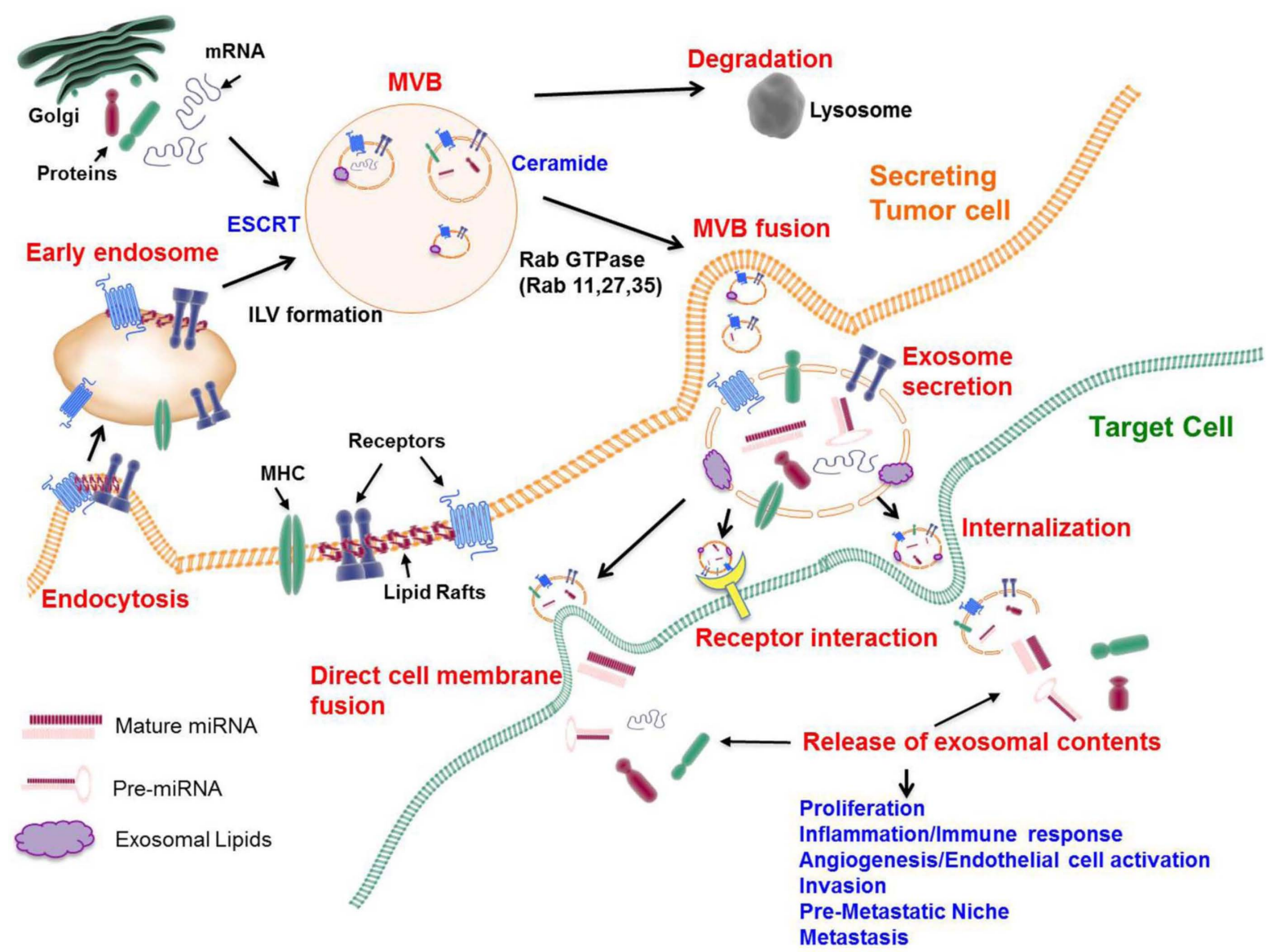

Fig. 1. Biogenesis, secretion, and intercellular communication of exosomes. Exosomes are formed as intraluminal vesicles (ILVs). Early endosomes are integrated into multivesicular bodies (MVBs), which are the source of exosomes through endosomal sorting complexes required for transport (ESCRT) machinery, lipids (such as ceramide), and the tetraspanins. During the process of ILV formation, proteins (membrane receptors, cytoplasmic proteins, tetraspanins), lipids (cholesterol, ceramide), and nucleic acids (DNA, mRNA, miRNA) are being incorporated into exosomes. MVBs fuse with lysosomes or with the cellular membrane to release exosomes into the extracellular space. Several Rab small GTPases (Rab 11, Rab 27, and Rab 35) are involved in transporting MVBs to the cell membrane and in exosome secretion. The uptake of exosomes into target cells is mediated by the fusion of exosomes with the cell membrane of target cells, by receptorligand interactions, or by endocytosis.

brane. ESCRT I and II induce membrane deformation into buds with sorted cargo, and ESCRT-III drives vesicle scission (10).

However, it should be noted that not all proteins are required for ubiquitination for their sorting into MVBs, and that ESCRT-independent mechanisms have been found to support that MVBs and ILVs can form in the absence of ESCRT function as inactivation of key proteins in the four different ESCRT complexes cannot block MVB formation (11). For example, tetraspanins can get into inwardly budding vesicles without ubiquitination owing to their intrinsic nature to partition into lipid raft-like subdomains of the plasma membrane (12). These subdomains are characterized by the lateral interaction between certain proteins and lipids and provide an important signaling function by placing signaling receptors in close prox- imity to promote their physical and functional interactions (13). In addition to these functions, lipid raft-like subdomains can generate membrane budding and fission by a ceramide-triggered process, suggesting an ESCRT-independent mechanism of MVB formation. It is not currently known whether mechanisms involving ESCRT, lipids, or tetraspanins act on the same MVB or on different MVBs (Fig. 1).

The subsets of ILVs within MVBs target cargo for lysosomal degradation in an ESCRT-dependent and lipid (ceramide)-independent manner. On the other hand, other subsets of ILVs release cargo to the cell's exterior in the form of exosomes, which appear to be ceramide biosynthesis-dependent and ESCRT-independent (Fig. 1). The latter process of directed transport involves multiple Rab family GTPases (Rab 11, Rab 27, 
and Rab 35) and SNARE family members (VAMP7 and YKT6) for targeted fusion with the plasma membrane (14). It remains to be investigated how cancer cells, as opposed to normal cells, package exosomes and regulate the sorting (degradation in the lysosome vs. release of exosomes) of vesicles within MVBs. Special sorting mechanisms that target tumor-suppressing elements containing ILVs to the lysosome, as well as direct oncogenic-elements containing ILVs to the plasma membrane, are probably present in invasive cancer cells. The tumor microenvironment may play a role in exosome secretion, since Rab GTPases 27a and 27b promote exosome secretion in response to hypoxia, which is frequently encountered in tumors (15).

Adhesion of exosomes to target cells depends upon transmembrane proteins expressed in exosomes and target cells. In cancer cells, the binding and uptake of exosomes to target cells involve a tetraspanin-integrin complex $(16,17)$. Recent evidence indicates that the integrin expression pattern in exosomes directs the sites of metastasis. For example, exosomal integrins $\alpha 6 \beta 4$ and $\alpha 6 \beta 1$ are associated with lung metastasis, whereas exosomal integrin $\alpha v \beta 3$ integrin is associated with liver metastasis (17). This study suggests that specific composition of exosomes contribute to organotropism associated with metastatic cancers. Uptake and internalization of exosomes to target cells can alter target cell functions by directly releasing their cargo into cytoplasm or by initiating juxtracrine signaling by means of receptor-ligand interactions $(18,19)$ (Fig. 1).

\section{ONCOGENIC ELEMENTS FOUND IN CCEs}

Cancer cells have been shown to produce excessive amounts of exosomes (referred to as cancer cell-derived exosomes (CCEs) via biological fluids. CCEs contain oncogenic elements including a variety of proteins, lipids, mRNAs, and microRNAs that can initiate the signaling pathways in tumor- and metastasis-promoting processes (20). The new techniques such as stable exosome purification, RNA sequencing, proteomics, and lipodomics have allowed cancer researchers to understand the mechanisms by which CCEs manipulate the local and distant environment to facilitate cancer growth and dissemination.

\section{Protein content}

Electron microscopic and proteomic analyses of exosomes reveal that the protein content of exosomes is derived mainly from endosomes, cytoplasm, and plasma membrane but not from other intracellular organelles, which suggests that exosomes represent endosomal compartments (21). Owing to their biogenesis through the endosomal network, exosomes contain specific proteins that are involved in intracellular membrane fusion and transport (Rab GTPases, annexins, and flotillin), tetraspanins (CD9, CD63, CD81, and CD82), heat shock proteins (Hsp60, Hsp70, and Hsp90), and proteins involved in MVB biogenesis (TSG101, Alix) $(4,17,21)$. They represent an evolutionarily conserved set of proteins that are commonly expressed in most exosomes, and such expression has been used to confirm the presence of exosomes in body fluids.

On the other hand, another group of unique exosomal proteins is specifically expressed in certain types of cells. These unique proteins are frequently found in CCEs depending on the cancer type and stage. Cancer-associated exosomal proteins play essential roles in the progression of these cancers by transferring their oncogenic, immunosuppressive, and metastatic functions to the primary tumor microenvironment or to metastatic niches. For example, glioma cells deliver the oncogenic mutant epidermal growth factor (EGF) receptor variant III (EGFRvIII) via exosomes to cells lacking EGFRvlll expression (22). The presence of EGFRvill confers on these cells the ability to become anti-apoptotic and to grow in an anchorage-independent manner (22). Anti-apoptotic and angiogenic mediators, such as vascular endothelial growth factor (VEGF), can be transferred to target cells to enhance the ability of recipient cells to morphologically transform (22). Mutant KRAS-expressing colon cancer cells transfer this constitutively active mutant oncogene into cells expressing wild-type KRAS via CCEs and increase the growth and tumorigenicity of recipient cells (23). CCEs can also confer drug resistance on recipient cells; for example, through the intercellular transfer of drug transporter MDR-1 via CCEs, docetaxel-resistant prostate cancer cells can induce chemoresistance in non-resistant prostate cancer cells (24). These findings nicely support the role of CCEs in increasing the tumorigenic potential and chemoresistance of recipient cells by malignant cancer cells.

CCEs secreted from the primary tumor play a role in establishing pre-metastatic niches that recruit metastasizing tumor cells and provide the environment for their subsequent growth. Highly metastatic melanoma-driven exosomes are able to convert bone marrow progenitors to a pro-metastatic phenotype through the hepatocyte growth factor (HGF) receptor, MET oncoprotein (25). CCEs from melanoma cells induce vascular leakiness at pre-metastatic sites and prepare sentinel lymph nodes for melanoma recruitment, extracellular matrix deposition, and vascular proliferation (26). CCEs are involved in tumorstromal interaction by transferring extracellular matrix metalloproteinase inducer (EMMPRIN) (also known as basigin and CD147) from tumor cells to fibroblasts (27). EMMPRIN stimulates the expression of metalloproteinase (MMP-1) in fibroblasts and the remodeling of the extracellular matrix (27). Exosomes secreted from fibroblasts could also stimulate breast cancer cell motility and invasion by stromal mobilization of the Wnt-PCP signaling pathway (28).

Evading immune response is one of the important hallmarks of cancer progression. There is considerable evidence to suggest that CCEs suppress tumor-specific immune responses in the host $(29,30)$. This somewhat contradicts previous reports that MHC class I molecules are present on the surface of exosomes secreted from virtually all cell types and can induce 
CD8-positive T-cell activation $(9,31)$. In addition, CCEs can be the source for tumor antigens that lead to activation of antigen-presenting cells and cytotoxic T lymphocytes $(32,33)$. However, cancer cells evolved the CCE-dependent strategies to avoid being recognized by the immune system. For example, Fas-L-, TRAIL-, or galectin-9-bearing CCEs can induce apoptosis of T lymphocytes $(29,30,34)$. The presence of prostaglandin E2 (PGE2) and transforming growth factor beta (TGF- $\beta$ ) in CCEs induces the accumulation of myeloid-derived suppressor cells (MDSCs) by switching the differentiation pathway of bone marrow myeloid cells to MDSCs (35). Accumulation of MDSCs leads to regulatory T-cell (Treg) expansion and apoptosis of tumor-reactive CD8-positive T lymphocytes (36). The results of these studies support the view that CCEs trigger immunosuppressive responses, which in turn favors the metastatic environment.

\section{Nucleic acid content}

Analysis of mRNA and miRNA profiles in CCEs and in parental cancer cells shows different patterns, suggesting a specific mechanism in the sorting of these nucleic acids into exosomes (37). It remains to be seen whether cancer cells develop the packing mechanisms that select oncogenic mRNAs and miRNAs (oncomiRs) over tumor-suppressive miRNAs into CCEs. In addition to packing of selective nucleic acids to CCEs, recent studies have demonstrated that CCEs in breast cancer cells contain the RISC-loading complex (Dicer, AGO2, and TRBP) that could process premature miRNAs into mature oncomiRs (38). These oncomiRs were shown to induce a neoplastic phenotype of targeted normal cells (38).

Studies of miRNA profiling have identified a group of exosomal miRNAs that are present at higher levels in CCEs than in normal exosomes. For example, four miRNAs (miR-1246, miR4644, miR-3976, and miR-4306) are more abundant in CCEs from the serum of patients with pancreatic cancer than in exosomes found in the serum from healthy donors (39). The levels of miR-200c and miR-214 are elevated in CCEs derived from patients with ovarian cancer and correlate with ovarian cancer stage (40). Another study showed that seven miRNAs (let-7a, miR-1229, miR-1246, miR-150, miR-21, miR-223, and miR23a) were significantly elevated in CCEs secreted from patients with primary colon cancer only at the early stage and were then decreased after surgical resection of the primary tumors, suggesting that the level of these miRNAs could be a useful tool for the early detection of colon cancer (41).

The transfer by CCEs of oncomiRs from the primary tumor or macrophages into recipient cells is associated with enhanced metastatic potential. Interleukin-4 (IL-4)-activated tumor-associated macrophages (TAMs) release exosomes that contain miR-223, a miRNA specific for IL-4-activated macrophages (42). Exosome-mediated delivery of miR-223 into co-cultured breast cancer cells enhanced their ability to invade (42). Gastric cancer tissue-derived mesenchymal stem cells (GC-MSCs) transfer exosomal miRNAs (miR-221/222) to gastric cancer cells, thus increasing gastric cancer cell migration (43). CCE-mediated secretion of miR-21 and miR-29a allows them to bind directly to toll-like receptors (TLRs) in immune cells, which triggers a TLR-mediated pro-metastatic response that leads to tumor growth and metastasis (44). In leukemia cells, an exosomal miR17-92 cluster consisting of six miRNAs (miR-17, -18a, -19a, $-20 a,-19 b$, and $-92 a)$ plays a role as communicator between cancer and endothelial cells to promote tumor angiogenesis (45). Exosomal miR105 secreted from metastatic breast cancer cells contributes to cancer metastasis by destroying the endothelial barriers through down-regulation of the tight junction protein ZO-1 (46). All this evidence suggests the efficacy of oncomiRs in CCEs in developing personalized diagnostics and therapeutics.

\section{Lipidomic content}

Exosomes have distinct lipid compositions in comparison to whole cell membranes. They are abundant in cholesterol, sphingomyelin, glycerophospholipids, phosphatidylserine, phosphatidylinositol, and ceramide (47). As for the connection between lipid dynamics and exosome biosynthesis, it has been reported that various lipids or lipid-related enzymes interact with proteins of the ESCRT machinery. For example, phosphatidylserine is required for the heat shock protein hsc-70-dependent microautophagy process generating exosomes (48). Lysobisphosphatidic acid association with the ESCRT III-associated protein Alix alters the dynamics of MVB and late endosome membrane $(49,50)$. Alix also binds to bismonoacylglycerolphosphate that is formed by phospholipase D2, an essential enzyme for exosome production (51). Independent of ESCRT protein machinery, the neutral lipid ceramide plays a key role in exosome biosynthesis by involving miRNA packaging (52). Cholesterol contributes to the secretion of flotilin-2-positive exosomes (53). In mesenchymal stem cells, sphingolipid cholesterol-rich microdomains (lipid rafts) are linked to the biogenesis and secretion of exosomes $(54,55)$.

How the lipid composition of CCEs differs from that of normal exosomes is not well understood. Combined approaches using shotgun lipidomics analyses of metastatic prostate cancer cells (PC3) and their exosomes revealed an overall 8.4-fold enrichment of lipids per mg of protein in CCEs (56). In this study, significant amounts of phosphatidylserine, cholesterol, sphingomyelin, and glycosphingolipids were found in exosomes, as compared with parental PC3 prostate cancer cells (56). These lipids could potentially be used as biomarkers of prostate cancer. In another study, shotgun lipidomic analysis of colorectal cancer cell line (LIM1215)-derived exosomes identified over 520 individual lipids (57). In a comparison with cellular lipid profiles, researchers observed the relative enrichment of exosomes with certain lipid classes, including sphingolipids, sterol lipids, glycerolipids, and glycerophospholipids (57). Most likely, the strategy of analysis used in this study can provide the basis for wide and comprehensive lipidome profiling of CCEs derived from other types of cancer cells and 
tissues.

Exosomal lipids can influence target cells by carrying lipid-related enzymes and bioactive lipids that influence signaling, inflammation, immunity, and their own biogenesis. More evidence suggests that lipids in CCEs can induce tumor growth and drug resistance. PGE2 is carried by CCEs and trigger host immunosuppression to allow tumor development (58). CCEs secreted from murine mammary adenocarcinoma cells contain PGE2 that is related to the induction of MDSC that down-regulate the functions of T cells (35). Function-blocking antibody against PGE2 blocks CCE-dependent MDSC induction and tumor growth (35). Exosomes from macrophages have been shown to contain enzymes for the biosynthesis of leukotrienes (LTs) that are potent pro-inflammatory lipid mediators (59). Receptors for LTB4 and LTC4 are overexpressed in some cancers, suggesting that exosomal LTs are probably involved in tumor development. Exosome biogenesis and secretion from B-cell lymphoma involve the phosphatidylcholine transporter ABCA3 (60). ABCA3 is implicated in shielding target cells from treatment with rituximab, an antibody that targets the B-cell lymphocyte antigen CD20 (60). Although ABCA3 is overexpressed in B-cell lymphomas, pharmacological inhibition or siRNA-mediated silencing of ABCA3 expression restores the susceptibility of target cells to antibody-mediated cell lysis, suggesting that ABCA3-dependent exosome release is implicated in resistance to immunotherapy (60).

\section{CLINICAL APPLICATIONS INVOLVING CCEs}

\section{Exosomes for cancer diagnosis and prognosis}

Circulating serum exosomes merit consideration as biomarkers for the early detection of cancer burden, because exosomes are stable at $4^{\circ} \mathrm{C}$ for 4 days and at $-70^{\circ} \mathrm{C}$ for long-term storage (40). The proteomics profiling of CCEs showed that cell-surface proteins in CCEs could be used as biomarkers for the diagnosis and prognosis of cancer. For example, Skog et al. demonstrated that the EGFRvIll mutant is expressed in a subtype of glioblastoma, and its expression is detected in serum circulating exosomes (61). This finding helped patients with glioblastoma to avoid invasive brain tumor biopsy, since determining EGFR status from blood samples will provide the information regarding the nature of this disease. In the colorectal cancer model, one study indicates that the level of circulating exosomes itself is related to poor prognosis and shorter survival of patients (62). Studies from immortalized hepatocellular carcinoma (HCC) revealed that CCEs could represent the cancer stage, since metastasis-inducing factors such as c-Met, caveolins, and S100 family members are present only in highly metastatic HCC-derived exosomes (63). Exosomes isolated from the blood of patients with breast cancer who had the worst prognosis showed metastatic and stemness-like signatures in the nucleic acids, suggesting that exosome-derived nucleic acids (mRNAs and miRNAs) can be used as suitable prognostic markers (64). Because the isolation of exosomes from patient samples is non-invasive and relatively easy, CCEs could emerge as the main source for diagnostic and prognostic markers of cancer, as long as cancer-specific markers in CCEs are successfully screened and identified.

\section{Therapeutic applications of exosomes}

Because exosomes represent a system that delivers selected cargo to target cells, they have great therapeutic potential (65). Therapeutic designs that block pathological intercellular transfer mediated by exosomes can prevent CCE-mediated tumor formation and metastasis. In this regard, the impairment of exosome secretion from cancer cells could be a potential target for cancer therapy. An increase in intracellular calcium levels stimulates exosome secretion (66). Blocking the function of $\mathrm{H}^{+} / \mathrm{NA}^{+}$and $\mathrm{Na}^{+} / \mathrm{Ca}^{+}$channels by means of dimethyl amirolide (DMA), an inhibitor of these two channels, effectively reduces exosome release in CT26-bearing mice, since these two channels regulate intracellular calcium levels (67). DMA also blocks the immunosuppressive function of bone marrow myeloid cells by inhibiting STAT3 phosphorylation (67). Another preclinical study involving the sphingomyelinase 2 inhibitor GW4869 showed that exosome-mediated tumor growth and metastasis are inhibited by this drug compound in lung cancer-bearing mice (44).

Alternatively, exosomes themselves can be used as delivery vesicles for therapeutic agents such as small interfering RNAs (siRNAs) or drug molecules that inhibit the functions of targeted cancer cells. Exosomes as a delivery tool merit consideration because they are not only bioavailable but also resistant to metabolic processes. Multiple studies support this possibility. Intravenous injection of exosomes carrying let-7a miRNA significantly reduced tumor formation in mice with EGFR-expressing breast cancer cells (68). Although the bioavailability and uptake of polyphenol compounds such as curcumin have been the major issue, curcumin becomes more stable and concentrated in blood once it is incorporated into exosomes (69). Clinical trials involving dendritic cell-derived exosomes for immunotherapy have been carried out $(70,71)$. Exosomes can be used for targeted drug delivery because they can lower immunogenicity and toxicity if they can selectively target cancer cells but not normal cells. For example, the fusion of integrin $\alpha v$-specific peptide to exosomal membrane protein led to highly efficient targeting of chemotherapeutics such as doxorubicin to integrin av-positive breast cancer cells and the inhibition of tumor growth in vivo without overt toxicity (72). Therefore, using exosomes as a drug carrier is likely to create a new opportunity for cancer treatment without side effects originating from off-target effects.

\section{CONCLUSIONS AND FUTURE DIRECTIONS}

Exosomes have emerged as a novel class of intercellular signal mediators that are involved in various pathological conditions, including cancer. In this review, we discussed the recent prog- 
ress in studies related to CCEs in terms of their oncogenic elements and therapeutic potentials. Compelling evidence from the recent literature supports the idea that CCEs can facilitate cancer invasion and metastasis by creating a fertile environment for tumor growth in the vicinity of neoplastic lesions as well as for distal metastases. The mechanisms by which CCEs achieve these effects include enhancement of the pro-tumorigenic potential of the recipient cells by introducing oncogenic elements and suppressing host immune responses. Exosomes also offer many novel therapeutic possibilities owing to their properties associated with easier detection and delivery potential. Advances in technology of exosome isolation, characterization, and in vitro modification allow selective delivery of the chosen exosome cargos to target cells. Detection of exosomes in bodily fluids represents a promising option for acquiring pathologic information for the diagnosis and prognosis of cancer. Future studies should focus on unresolved issues, such as determining the intricacies of CCE-specific biogenesis and sorting, enhancing the purity of exosome isolation, and developing safer routes of administration to achieve maximal targeted delivery of therapeutic exosomes.

\section{ACKNOWLEDGEMENTS}

This research was supported by grants from the American Cancer Society (RSG-09-091-01-CSM; Principal Investigator, Jun Chung) and the National Institutes of Health-National Cancer Institute (NIH-NCl) (R01CA163657-01A1; Principal Investigator, Jun Chung).

\section{REFERENCES}

1. Raposo G and Stoorvogel W (2013) Extracellular vesicles: exosomes, microvesicles, and friends. J Cell Biol 200, 373-383

2. EL Andaloussi S, Mäger I, Breakefield XO and Wood MJ (2013) Extracellular vesicles: biology and emerging therapeutic opportunities. Nat Rev Drug Discov 12, 347-357

3. Johnstone RM, Adam M, Hammond JR, Orr L and Turbide C (1987) Vesicle formation during reticulocyte maturation. Association of plasma membrane activities with released vesicles (exosomes). J Biol Chem 262, 9412-9420

4. Vlassov AV, Magdaleno S, Setterquist R and Conrad R (2012) Exosomes: Current knowledge of their composition, biological functions, and diagnostic and therapeutic potentials. Biochim Biophys Acta 1820, 940-948

5. Kharaziha P, Ceder S, Li Q and Panaretakis T (2012) Tumor cell-derived exosomes: A message in a bottle. Biochim Biophys Acta 1826, 103-111

6. Valadi H, Ekström K, Bossios A, Sjöstrand M, Lee JJ and Lötvall JO (2007) Exosome-mediated transfer of mRNAs and microRNAs is a novel mechanism of genetic exchange between cells. Nat Cell Biol 9, 654-659

7. Milane L, Singh A, Mattheolabakis G, Suresh M and Amiji MM (2015) Exosome mediated communication within the tumor microenvironment. J Control Release 219, 278-294

8. Théry C, Zitvogel L and Amigorena S (2002) Exosomes: composition, biogenesis and function. Nat Rev Immunol 2, 569-579

9. Robbins PD and Morelli AE (2014) Regulation of immune responses by extracellular vesicles. Nat Rev Immunol 14, 195-208

10. Hanson PI and Cashikar A (2012) Multivesicular body morphogenesis. Annu Rev Cell Dev Biol 28, 337-362

11. Stuffers S, Sem Wegner C, Stenmark H, Brech A (2009) Multivesicular Endosome Biogenesis in the Absence of ESCRTs. Traffic 10, 925-937

12. Hemler ME (2001) Specific tetraspanin functions. J Cell Biol 155, 1103-1107

13. Edidin M (2001) Shrinking patches and slippery rafts: scales of domains in the plasma membrane. Trends Cell Biol 11, 492-496

14. Pant S, Hilton $H$ and Burczynski ME (2012) The multifaceted exosome: biogenesis, role in normal and aberrant cellular function, and frontiers for pharmacological and biomarker opportunities. Biochem Pharmacol 83, 1484-1494

15. King HW, Michael MZ and Gleadle JM (2012) Hypoxic enhancement of exosome release by breast cancer cells. BMC Cancer 12, 421

16. Nazarenko I, Rana S, Baumann A et al (2010) Cell surface tetraspanin Tspan8 contributes to molecular pathways of exosome-induced endothelial cell activation. Cancer Res 70, 1668-1678

17. Hoshino A, Costa-Silva B, Shen T-L et al (2015) Tumour exosome integrins determine organotropic metastasis. Nature 28, 15756

18. Blanchard N, Lankar D, Faure F et al (2002) TCR activation of human $T$ cells induces the production of exosomes bearing the TCR/CD3/zeta complex. J Immunol Baltim Md 168, 3235-3241

19. Montecalvo A, Larregina AT, Shufesky WJ et al (2012) Mechanism of transfer of functional microRNAs between mouse dendritic cells via exosomes. Blood 119, 756-766

20. Kahlert C and Kalluri R (2013) Exosomes in tumor microenvironment influence cancer progression and metastasis. J Mol Med 91, 431-437

21. Théry C, Boussac M, Véron P et al (2001) Proteomic analysis of dendritic cell-derived exosomes: a secreted subcellular compartment distinct from apoptotic vesicles. J Immunol Baltim Md 166, 7309-7318.

22. Al-Nedawi K, Meehan B, Micallef J et al (2008) Intercellular transfer of the oncogenic receptor EGFRvIII by microvesicles derived from tumour cells. Nat Cell Biol 10, 619-624

23. Demory Beckler M, Higginbotham JN, Franklin JL et al (2013) Proteomic analysis of exosomes from mutant KRAS colon cancer cells identifies intercellular transfer of mutant KRAS. Mol Cell Proteomics 12, 343-355

24. Corcoran C, Rani S, O'Brien K et al (2012) Docetaxel-resistance in prostate cancer: evaluating associated phenotypic changes and potential for resistance transfer via exosomes. PloS One 7, e50999

25. Peinado H, Alečković M, Lavotshkin S et al (2012) Melanoma exosomes educate bone marrow progenitor cells toward a pro-metastatic phenotype through MET. Nat Med $18,883-891$ 
26. Hood JL, San RS and Wickline SA (2011) Exosomes released by melanoma cells prepare sentinel lymph nodes for tumor metastasis. Cancer Res 71, 3792-3801

27. Sidhu SS, Mengistab AT, Tauscher AN, LaVail J and Basbaum C (2004) The microvesicle as a vehicle for EMMPRIN in tumor-stromal interactions. Oncogene 23, 956-963

28. Luga V and Wrana JL (2013) Tumor-stroma interaction: Revealing fibroblast-secreted exosomes as potent regulators of Wnt-planar cell polarity signaling in cancer metastasis. Cancer Res 73, 6843-6847

29. Huber V, Fais $S$, lero $M$ et al (2005) Human colorectal cancer cells induce T-cell death through release of proapoptotic microvesicles: role in immune escape. Gastroenterology 128, 1796-1804.

30. Klibi J, Niki T, Riedel A et al (2009) Blood diffusion and Th1-suppressive effects of galectin-9-containing exosomes released by Epstein-Barr virus-infected nasopharyngeal carcinoma cells. Blood 113, 1957-1966

31. Théry C, Ostrowski M and Segura E (2009) Membrane vesicles as conveyors of immune responses. Nat Rev Immunol 9, 581-593

32. Wolfers J, Lozier A, Raposo G et al (2001) Tumor-derived exosomes are a source of shared tumor rejection antigens for CTL cross-priming. Nat Med 7, 297-303

33. Andre F, Schartz NEC, Movassagh M et al (2002) Malignant effusions and immunogenic tumour-derived exosomes. Lancet 360, 295-305

34. Andreola G, Rivoltini L, Castelli C et al (2002) Induction of lymphocyte apoptosis by tumor cell secretion of FasL-bearing microvesicles. J Exp Med 195, 1303-1316

35. Xiang X, Poliakov A, Liu C et al (2009) Induction of myeloid-derived suppressor cells by tumor exosomes. Int J Cancer 124, 2621-2633

36. Wieckowski EU, Visus C, Szajnik M, Szczepanski MJ, Storkus WJ and Whiteside TL (2009) Tumor-derived microvesicles promote regulatory $\mathrm{T}$ cell expansion and induce apoptosis in tumor-reactive activated CD8 + T lymphocytes. J Immunol 183, 3720-3730

37. Ohshima K, Inoue K, Fujiwara A et al (2010) Let-7 microRNA family is selectively secreted into the extracellular environment via exosomes in a metastatic gastric cancer cell line. PloS One 5, e13247

38. Anastasiadou E and Slack FJ (2014) Malicious exosomes. Science 346, 1459-1460

39. Madhavan B, Yue S, Galli U et al (2015) Combined evaluation of a panel of protein and miRNA serum-exosome biomarkers for pancreatic cancer diagnosis increases sensitivity and specificity. Int J Cancer 136, 2616-2627

40. Taylor DD and Gercel-Taylor C (2008) MicroRNA signatures of tumor-derived exosomes as diagnostic biomarkers of ovarian cancer. Gynecol Oncol 110, 13-21

41. Ogata-Kawata H, Izumiya M, Kurioka D et al (2014) Circulating Exosomal microRNAs as Biomarkers of Colon Cancer. PLoS One 9, e92921

42. Yang M, Chen J, Su F et al (2011) Microvesicles secreted by macrophages shuttle invasion-potentiating microRNAs into breast cancer cells. Mol Cancer 10, 117

43. Wang M, Zhao C, Shi H et al (2014) Deregulated microRNAs in gastric cancer tissue-derived mesenchymal stem cells: novel biomarkers and a mechanism for gastric cancer. Br J Cancer 110, 1199-1210

44. Fabbri M, Paone A, Calore F et al (2012) MicroRNAs bind to Toll-like receptors to induce prometastatic inflammatory response. Proc Natl Acad Sci U S A 109, E2110-2116

45. Umezu T, Ohyashiki K, Kuroda $M$ and Ohyashiki JH (2013) Leukemia cell to endothelial cell communication via exosomal miRNAs. Oncogene 32, 2747-2755

46. Zhou W, Fong MY, Min Y et al (2014) Cancer-Secreted miR-105 Destroys Vascular Endothelial Barriers to Promote Metastasis. Cancer Cell 25, 501-515

47. Record M, Carayon K, Poirot M and Silvente-Poirot S (2014) Exosomes as new vesicular lipid transporters involved in cell-cell communication and various pathophysiologies. Biochim Biophys Acta 184, 108-120

48. Sahu R, Kaushik S, Clement CC et al (2011) Microautophagy of cytosolic proteins by late endosomes. Dev Cell 20, 131-139

49. Dikic I (2004) ALIX-ing phospholipids with endosome biogenesis. BioEssays 26, 604-607

50. Matsuo H, Chevallier J, Mayran N et al (2004) Role of LBPA and Alix in Multivesicular Liposome Formation and Endosome Organization. Science 303, 531-534

51. Laulagnier K, Grand D, Dujardin A et al (2004) PLD2 is enriched on exosomes and its activity is correlated to the release of exosomes. FEBS Lett 572, 11-14

52. Trajkovic K, Hsu C, Chiantia S et al (2008) Ceramide triggers budding of exosome vesicles into multivesicular endosomes. Science 319, 1244-1247

53. Strauss K, Goebel C, Runz H et al (2010) Exosome Secretion Ameliorates Lysosomal Storage of Cholesterol in Niemann-Pick Type C Disease. J Biol Chem 285, 2627926288

54. Ikonen E (2001) Roles of lipid rafts in membrane transport. Curr Opin Cell Biol 13, 470-477

55. Tan SS, Yin Y, Lee T et al (2013) Therapeutic MSC exosomes are derived from lipid raft microdomains in the plasma membrane. J Extracell Vesicles 2, 22614

56. Llorente A, Skotland T, Sylvänne T et al (2013) Molecular lipidomics of exosomes released by PC-3 prostate cancer cells. Biochim Biophys Acta 1831, 1302-1309

57. Lydic TA, Townsend S, Adda CG et al (2015) Rapid and comprehensive "shotgun" lipidome profiling of colorectal cancer cell derived exosomes. Methods 4, 014

58. Wang D and DuBois RN (2010) Eicosanoids and cancer. Nat Rev Cancer 10, 181-193

59. Esser J, Gehrmann U, D'Alexandri FL et al (2010) Exosomes from human macrophages and dendritic cells contain enzymes for leukotriene biosynthesis and promote granulocyte migration. J Allergy Clin Immunol 126, 1032-1040

60. Aung T, Chapuy B, Vogel D et al (2011) Exosomal evasion of humoral immunotherapy in aggressive B-cell lymphoma modulated by ATP-binding cassette transporter A3. Proc Natl Acad Sci U S A 108, 15336-15341

61. Skog J, Würdinger T, van Rijn S et al (2008) Glioblastoma microvesicles transport RNA and proteins that promote tumour growth and provide diagnostic biomarkers. Nat Cell Biol 10, 1470-1476

62. Silva J, Garcia V, Rodriguez M et al (2012) Analysis of 
exosome release and its prognostic value in human colorectal cancer. Genes Chromosomes Cancer 51, 409-418

63. He M, Qin H, Poon TCW et al (2015) Hepatocellular carcinoma-derived exosomes promote motility of immortalized hepatocyte through transfer of oncogenic proteins and RNAs. Carcinogenesis 36, 1008-18

64. Rodríguez M, Silva J, Herrera A et al (2015) Exosomes enriched in stemness/metastatic-related mRNAS promote oncogenic potential in breast cancer. Oncotarget 6, 40575-40587

65. Merino AM, Hoogduijn MJ, Borras FE and Franquesa M (2014) Therapeutic potential of extracellular vesicles. Front Immunol 5, 658

66. Savina A, Furlán M, Vidal M, Colombo MI (2003) Exosome Release Is Regulated by a Calcium-dependent Mechanism in K562 Cells. J Biol Chem 278, 2008320090

67. Chalmin F, Ladoire S, Mignot G et al (2010) Membrane-associated Hsp72 from tumor-derived exosomes mediates STAT3-dependent immunosuppressive function of mouse and human myeloid-derived suppressor cells. J Clin Invest 120, 457-471

68. Ohno S, Takanashi M, Sudo K et al (2013) Systemically injected exosomes targeted to EGFR deliver antitumor microRNA to breast cancer cells. Mol Ther 21, 185-191

69. Sun D, Zhuang X, Xiang X et al (2010) A novel nanoparticle drug delivery system: the anti-inflammatory activity of curcumin is enhanced when encapsulated in exosomes. Mol Ther 18, 1606-1614

70. Escudier B, Dorval T, Chaput $\mathrm{N}$ et al (2005) Vaccination of metastatic melanoma patients with autologous dendritic cell (DC) derived-exosomes: results of thefirst phase I clinical trial. J Transl Med 3, 10

71. Pitt JM, Charrier M, Viaud S et al (2014) Dendritic cell-derived exosomes as immunotherapies in the fight against cancer. J Immunol 193, 1006-1011

72. Tian Y, Li S, Song J et al (2014) A doxorubicin delivery platform using engineered natural membrane vesicle exosomes for targeted tumor therapy. Biomaterials 35, 23832390 